\title{
Calculating predictive values for the large repeat alleles at the SCA8 locus in patients with ataxia
}

\author{
V Juvonen, V Kairisto, M Hietala, M-L Savontaus
}

J Med Genet 2002;39:935-936

S nce the original description of SCA8 in 1999, ${ }^{1}$ several reports have been published on the occurrence of the SCA8 gene CTG repeat expansion in various populations. These studies have shown that, in addition to finding the SCA8 expansion in familial and sporadic ataxia patients, expanded alleles can also be found in non-ataxic subjects, psychiatric patients, and in patients with various other neurological diseases with a known aetiological cause..$^{2-6}$ Owing to the exceptionally low penetrance of the mutation, questions have been raised about the pathogenic role of the expansion, and there exists speculation about alternative mechanisms, such as linkage disequilibrium of the CTG expansion with another as yet unidentified causal mutation. Accordingly, caution in interpreting mutation findings in clinical samples has been stressed and some investigators discourage genetic testing for SCA8 until a pathological mechanism has been established. ${ }^{3}{ }^{6}$

\section{SUBJECTS AND METHODS}

We have investigated the occurrence of the SCA8 repeat expansion in 251 unrelated Finnish SCA patients and found 22 of them with the expansion. Based on our present and previous studies on controls and patients, we define the size range of 15-40 combined repeats as normal alleles and above 80 up to 800 as expanded alleles. Intermediate alleles between these groups are sometimes seen and we interpret them to be most likely non-pathogenic. We used a combination of PCR and Southern blotting methods as described previously ${ }^{5}$ in order to reach $100 \%$ sensitivity for the detection of expansions. None of the 22 SCA 8 positive patients had expansions at the SCAl, 2 , 3, 6, 7, 10, 12, 17, DRPLA, or FRDA loci. Thirteen of the SCA8 expansion positive patients had a family history of the disease and, in nine of them, it was compatible with a dominant inheritance pattern.

\section{RESULTS AND DISCUSSION}

The number of combined CTA/CTG repeats in the expanded alleles of the hereditary cases were 97, 118, 119, 121, 125, 128, $128,151,151,196,199,345$, and 660 . Among the nine sporadic patients with expansions, three had expanded repeats on both

\section{Key points}

- We report spinocerebellar ataxia 8 (SCA8) repeat expansion data in 251 unrelated Finnish SCA patients.

- SCA8 repeat expansion was significantly associated with ataxia but the clinical specificity for the repeat expansion test is no higher than $97.1 \%$.

- For non-symptomatic subjects with a low pre-test probability for SCA8, virtually none of the positive results are correlated with the risk of ataxia, and the predictive value of the finding is negligible.

- However, the test can be considered as a helpful diagnostic tool with a predictive value of $68 \%$ when evaluating patients with symptoms of SCA. A positive family history further increases the predictive value of the test to above $80 \%$.

alleles (101/156, 105/137, 134/151, 115, 168, 204, 504, 620, and 800 combined repeats on expanded alleles). Table 1 gives the frequencies of the SCA8 expansions and the respective 95\% confidence intervals calculated using Freeman and Tukey's approximation in the different subgroups. The frequency of subjects with SCA8 expansions was statistically significantly $(p<0.002)$ higher in hereditary ataxias than among the 448 non-ataxic controls studied previously. ${ }^{5}$ The assumption ${ }^{6}$ that SCA8 has a prevalence of approximately $1 / 100000$ gives a penetrance of only $0.03 \%$ for the SCA8 expansion in Finland. It is obvious that genetic testing for SCA8 in clinical samples cannot be diagnostic on these premises.

However, as seen in table 1 , the Finnish series shows that SCA8 expansions cluster in ataxia patients and this clustering is more conspicuous and statistically significant in the hereditary cases. Calculation of predictive values (PV), that is, the probability of disease after getting a positive test result, for each group of patients with ataxia symptoms and a defined family history of the disease, could thus be clinically important. However, the test should not be applied to non-symptomatic relatives of patients with ataxia.

\begin{tabular}{|c|c|c|c|c|}
\hline & No of subjects & $\begin{array}{l}\text { Subjects with } \\
\text { SCA8 } \\
\text { expansion }\end{array}$ & $\begin{array}{l}\text { SCA8 } \\
\text { expansion } \\
\text { frequency }\end{array}$ & $\begin{array}{l}95 \% \text { confidence } \\
\text { interval for frequency }\end{array}$ \\
\hline All ataxia patients & 251 & 22 & $8.8 \% *$ & $5.6 \%$ to $13.0 \%$ \\
\hline Hereditary ataxia & 86 & 13 & $15.1 \% *$ & $9.2 \%$ to $23.1 \%$ \\
\hline Dominant ataxia & 49 & 9 & $18.4 \% *$ & $10.0 \%$ to $30.1 \%$ \\
\hline Sporadic ataxia & 165 & 9 & $5.5 \% \dagger$ & $2.9 \%$ to $9.4 \%$ \\
\hline Non-ataxic controls ${ }^{5}$ & 448 & 13 & $2.9 \%$ & $1.7 \%$ to $4.6 \%$ \\
\hline
\end{tabular}

${ }^{*} p<0.002$ when the frequency is compared to non-ataxic controls by chi-square statistic with Yates's continuity correction ( $\dagger$ not significant). 


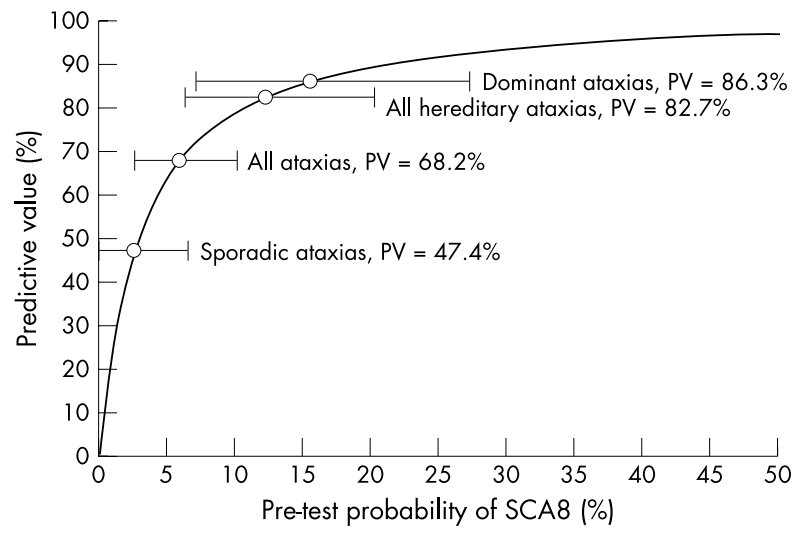

Figure 1 Predictive value (PV) of the SCA8 expansion finding as a function of the pre-test probability of SCA8. Horizontal bars show the 95\% confidence limits for pre-test probability.

The concept of likelihood ratio (LR) is useful to derive predictive values (PV) for a disease from the estimated pre-test probability (Pr). It shows how much more probable it is for a diseased subject to obtain a true positive diagnostic test result than for a healthy person to get a false positive diagnostic test result. The likelihood ratio itself is not dependent on the prevalence of the disease. If clinical sensitivity (Se) and specificity (Sp) are known, the likelihood ratio can be calculated $^{78}$ using the equation $\mathrm{LR}=\mathrm{Se} /(\mathrm{l}-\mathrm{Sp})$. For SCA8, the clinical sensitivity can be assumed to be $\sim 100 \%$ since all "true" SCA8 patients by definition have the expansion. Clinical specificity is the probability of a non-ataxic subject obtaining a negative result (in our material 97.1\%). This gives a likelihood ratio of 34.5 for SCA8 testing in the Finnish material.

The frequencies in table 1 , after the expected proportion of false positive findings has been subtracted, can be considered to give the pre-test probability (Pr) of SCA8 ataxia in each representative subgroup. If the false positive rate for SCA8 among ataxia patients is assumed to be the same as in non-ataxic controls $(2.9 \%)$, the following pre-test probabilities will be obtained: $5.9 \%$ for all ataxia patients, $12.2 \%$ for all inherited ataxia patients, $15.5 \%$ for the dominant inheritance pattern group, and $2.6 \%$ for sporadic ataxia.

When pre-test probability (Pr) and likelihood ratio (LR) are known, the predictive value (PV) can be calculated according to the following formula :

$\mathrm{PV}=(\mathrm{Pr} \times \mathrm{LR}) /((\mathrm{l}-\mathrm{Pr})+(\mathrm{Pr} \times \mathrm{LR}))$

As the likelihood ratio can be considered to be constant (34.5 in the Finnish material), predictive value is solely dependent, in a non-linear fashion, on pre-test probability. For the different ataxia patient groups in this study, the predictive values of a positive SCA8 repeat expansion result are the following: all ataxia patients $68.2 \%$, inherited ataxia $82.7 \%$, dominant inheritance pattern $86.3 \%$, and sporadic ataxia $47.4 \%$ (fig 1). For a non-ataxic control, the predictive value for SCA8 is negligible.

It has been shown that factors such as repeat length affect disease penetrance. ${ }^{9}$ Moreover, the unusual features of the repeat tract such as the polymorphic length of the CTA sequence as well as interruptions within the CTG repeat could be factors that affect penetrance. Unfortunately, the sample size of our study was not sufficient to make statistical calculations on different expansion subclasses. However, we think it will be advantageous to include all these factors in calculations of penetrance and predictive values as soon as a sufficient amount of data is available. Given the relatively high frequency of SCA8 repeat expansions in non-ataxic subjects in Finland, homozygous expansion carriers are expected at about a frequency of $0.1 \%$. It is noteworthy that we found three among our nine sporadic ataxia patients. This may suggest that homozygosity increases the penetrance.

In comparison to many other populations, Finnish nonataxic controls show an unusually high frequency of the SCA8 repeat expansion..$^{1-6}$ Lower frequencies would result in a better likelihood ratio for SCA8 testing and an upward shift of the curve in fig l. Thus, in other populations an even lower pre-test probability of SCA8 could result in a relatively high predictive value.

Despite its well established status in clinical chemistry, ${ }^{8}$ calculation of predictive values is not widely recognised or commonly used in genetic testing. Besides SCA8, it could, however, be applied to many genetic analyses with disease association.

\section{Authors' affiliations}

V Juvonen, M-L Savontaus, Department of Medical Genetics, University of Turku, Finland

V Juvonen, V Kairisto, Turku University Hospital Laboratories, Turku, Finland

V Kairisto, Department of Clinical Chemistry, University of Turku, Finland M Hietala, Clinical Genetics Unit, Turku University Hospital, Finland M-L Savontaus, Department of Biology, University of Turku, Finland

Correspondence to: Dr V Juvonen, Turku University Hospital, Laboratory Department 931, Kiinamyllynkatu 4-8, FIN-20520 Turku, Finland; vesa.juvonen@tyrs.fi

\section{REFERENCES}

1 Koob MD, Moseley ML, Schut L, Benzow KA, Bird TD, Day JW, Ranum LP. An untranslated CTG expansion causes a novel form of spinocerebellar ataxia (SCA8). Nat Genet 1999;21:379-84.

2 Stevanin G, Herman A, Durr A, Jodice C, Frontali M, Agid Y, Brice A. Are (CTG)n expansions at the SCA8 locus rare polymorphisms? Nat Genet 2000:24:213.

3 Worth PF, Houlden H, Giunti P, Davis MB, Wood NW. Large, expanded repeats in SCA8 are not confined to patients with cerebellar ataxia. Nat Genet 2000;24:214-15.

4 Vincent JB, Neves-Pereira ML, Paterson AD, Yamamoto E, Parikh SV, Macciardi F, Gurling HM, Potkin SG, Pato CN, Macedo A, Kovacs M, Davies M, Lieberman JA, Meltzer HY, Petronis A, Kennedy JL. An unstable trinucleotide-repeat region on chromosome 13 implicated in spinocerebellar ataxia: a common expansion locus. Am J Hum Genet 2000:66:819-29.

5 Juvonen V, Hietala $M$, Paivarinta $M$, Rantamaki $M$, Hakamies $L$, Kaakkola S, Vierimaa O, Penttinen M, Savontaus ML. Clinical and genetic findings in Finnish ataxia patients with the spinocerebellar ataxia 8 repeat expansion. Ann Neurol 2000;48:354-61.

6 Sobrido MJ, Cholfin JA, Perlman S, Pulst SM, Geschwind DH. SCA8 repeat expansions in ataxia: a controversial association. Neurology 2001;57:1310-12.

7 Albert A. On the use and computation of likelihood ratios in clinical chemistry. Clin Chem 1982;28:1113-19.

8 Halkin A, Reichman M, Schwaber O, Paltiel O, Brezis M. Likelihood ratios: getting diagnostic testing into perspective. Q J Med 1998;91:247-58

9 Day JW, Schut L, Moseley ML, Durand AC, Ranum LP. Spinocerebellar ataxia type 8 . Clinical features in a large family. Neurology 2000;55:649-57. 